\title{
Direct Oral Anticoagulants versus Vitamin K Antagonists in epistaxis patients: a systematic review and meta-analysis
}

\author{
Petar Stanković ${ }^{1}$, Stephan Hoch ${ }^{2}$, Stefan Rudhart ${ }^{2}$, Danilo Obradovic ${ }^{3}$, Nikolaos Dagres ${ }^{3}$, \\ and Thomas Wilhelm ${ }^{1}$ \\ ${ }^{1}$ Sana Kliniken Leipziger Land GmbH \\ ${ }^{2}$ Philipp University \\ ${ }^{3}$ Leipzig Heart Centre University Hospital Clinic for Cardiology
}

September 26, 2021

\begin{abstract}
Objective: Epistaxis is the most common otolaryngological emergency and up to one third of patients in treated on an inpatient basis take oral anticoagulants (OAC). Direct oral anticoagulants (DOAC), an OAC subgroup, have been on the market since 2010 and are being increasingly prescribed due to the cardiologic and hematologic guidelines that favour them over vitamin $\mathrm{K}$ antagonists (VKA), the older of the OAC subgroups. The present study aims to investigate which subgroup of epistaxis patients taking OACs has a more favourable outcome. Design/Setting: A systematic review and meta-analysis were performed according to the PRISMA 2020 statement using the PubMed and Cochrane Library databases. Continuous data was analysed and standardized mean difference (SMD) was calculated according to Hedges' g. Dichotomous data was analysed and the Mantel-Haenszel method was applied to establish the odds ratio (OR). Heterogeneity was assessed according to the I2 statistics. Main Outcome/Results: A total of 8 reports covering 1390 patients were included in the final synthesis. The pooled analysis demonstrated significantly shorter hospital stays in the DOAC group ( $\mathrm{SMD}=-0.22,95 \% \mathrm{CI}-0.42$ to $-0.02, \mathrm{P}=.03$ ) and a significantly higher rate of posterior bleeding in the VKA group $(\mathrm{OR}=.39,95 \%$ CI .23 to $.68, \mathrm{P}=.001)$. No statistically significant differences with regard to recurrence rates, admission rates, the need for transfusion, or surgical intervention $(\mathrm{P}=$ $.57, .12, .57$ and .38 respectively) were found. Conclusion: According to this meta-analysis, epistaxis patients taking DOACs have a more favourable outcome than patients taking VKAs.
\end{abstract}

\begin{abstract}
Objective : Epistaxis is the most common otolaryngological emergency and up to one third of patients in treated on an inpatient basis take oral anticoagulants (OAC). Direct oral anticoagulants (DOAC), an OAC subgroup, have been on the market since 2010 and are being increasingly prescribed due to the cardiologic and hematologic guidelines that favour them over vitamin $\mathrm{K}$ antagonists (VKA), the older of the OAC subgroups. The present study aims to investigate which subgroup of epistaxis patients taking OACs has a more favourable outcome.

Design/Setting : A systematic review and meta-analysis were performed according to the PRISMA 2020 statement using the PubMed and Cochrane Library databases. Continuous data was analysed and standardized mean difference (SMD) was calculated according to Hedges' g. Dichotomous data was analysed and the Mantel-Haenszel method was applied to establish the odds ratio (OR). Heterogeneity was assessed according to the $\mathrm{I}^{2}$ statistics.
\end{abstract}

Main Outcome/Results : A total of 8 reports covering 1390 patients were included in the final synthesis. The pooled analysis demonstrated significantly shorter hospital stays in the DOAC group ( $\mathrm{SMD}=-0.22$, $95 \%$ CI -0.42 to $-0.02, \mathrm{P}=.03)$ and a significantly higher rate of posterior bleeding in the VKA group $(\mathrm{OR}=$ 
$.39,95 \%$ CI .23 to $.68, \mathrm{P}=.001)$. No statistically significant differences with regard to recurrence rates, admission rates, the need for transfusion, or surgical intervention $(\mathrm{P}=.57, .12, .57$ and .38 respectively) were found.

Conclusion : According to this meta-analysis, epistaxis patients taking DOACs have a more favourable outcome than patients taking VKAs.

Keywords: anticoagulants, epistaxis, meta-analysis, doac, vka

\section{Key points}

- Almost one third of inpatient epistaxis patients take oral anticoagulants (OAC) with an upward trend. These are either vitamin K antagonists (VKA) or direct anticoagulants (DOAC).

- The proportion of DOAC epistaxis patients is continually rising.

- Epistaxis patients taking DOACs have a more favourable outcome compared to patients taking VKAs because they spend less time in hospital and are more likely to have anterior than posterior epistaxis, which is easier to control.

- No significant differences exist between the two groups with regard to recurrence rates, admission rates, the need for transfusion, or surgical intervention.

\section{Objective}

Epistaxis is the most common emergency in otolaryngology ${ }^{1,2}$, accounting for up to one third of all emergencies ${ }^{3,4}$. The treatment is discomforting and has a huge socio-economic impact ${ }^{5,6}$. Up to one third of all epistaxis patients in inpatient treatment are taking oral anticoagulants $(\mathrm{OAC})^{7-9}$. A steep rising trend in this subgroup of epistaxis patients has been noted over the past several years ${ }^{10}$. As the general population of the industrialized countries becomes older, this tendency is expected to continue.

Until 2010, OACs were almost exclusively vitamin K inhibitors (VKA). However in the years following, a new generation of OACs, direct oral anticoagulants (DOAC), was introduced to the market. These drugs are Factor Xa or IIa inhibitors and are more easily administered as they do not require pharmacokinetics monitoring with dose adjustment ${ }^{11}$. The current cardiology and haematology guidelines favour DOACs over VKAs in the prevention of thromboembolic events in patients with atrial fibrillation ${ }^{11}$ and venous thromboembolism ${ }^{12}$. This has created a shift towards more DOAC patients in everyday practice, a shift which has also been confirmed and reported on in the most recent papers on epistaxis patients on OACs in the ENT emergency room ${ }^{7,13}$.

The purpose of the present study is to compare the outcomes of epistaxis patients taking DOACs and VKAs using a qualitative and quantitative synthesis of the available literature.

\section{Design/Setting/Main Outcome}

\subsection{Literature search}

The systematic review was done in accordance with the PRISMA $2020^{14}$ statement. Two authors (PS and TW) searched independently on PubMed and the Cochrane database on April $25^{\text {th }} 2021$ using the following criteria: (epistaxis AND anticoagulation) OR (epistaxis AND DOAC). The search was restricted to the years following the FDA approval ${ }^{15}$ of the first DOAC (2009-2021). Disagreements were settled through consensus after thorough analysis. Additionally, the references of reviewed papers were also subject to analysis. No automatization tools were used in the process.

\subsection{Study selection, bias assessment and data retrieval}

The inclusion criterion was two-armed studies (DOAC vs. VKA) on consecutive epistaxis patients with a minimum of 20 patients in each group. The exclusion criteria were absence of a thorough comparison between DOAC and VKA groups, group size of $<20$ patients, duplicate studies, case reports, reviews, comments, animal studies, and letters to the editor. Data extraction was performed by two authors (PS 
and TW) independently. All studies were assessed for bias using the MINORS ${ }^{16}$ score for comparative non-randomized studies. Discrepancies were settled after a consensus between all authors had been reached.

\subsection{Outcomes}

The following outcomes were extracted: total number of epistaxis patients taking DOACs and VKAs, admission rates, days in hospital, posterior epistaxis, interventions needed, transfusions, recurrence rates, and haemoglobin values.

\subsection{Statistical analysis}

The meta-analysis was performed using the MedCalc Statistical Software version 17.8.6 (MedCalc Software bvba, Ostend, Belgium; http://www.medcalc.org; 2017). A standardized mean difference (SMD) with a 95\% confidence interval (CI) and a pooled standard deviation (SE) calculated according to Hedges' g was used to compare the days in hospital and haemoglobin values. Odds ratios (OR) with $95 \%$ CI according to the Mantel-Haenszel method were used to compare the rates of admission, posterior epistaxis, interventions, transfusions, and recurrence. Heterogeneity was accessed according to $\mathrm{I}^{2}$ statistics. When the $\mathrm{I}^{2}$ statistics did not reach statistical significance, the fixed effects model was used; otherwise the random effects model was applied. Forest and funnel plots were created for each investigated result. A two sided $\mathrm{P}$ value $<.05$ was considered statistically significant.

\section{Results}

\subsection{Study selection and characteristics}

A total of 286 records were screened using the aforementioned search criteria. After excluding 9 duplicates, we further excluded 250 records based on title and abstract, resulting in 27 studies for full text review. After the additional exclusion of 19 studies that did not meet the inclusion criterion (16 studies were one arm or didn't have a thorough comparison between the groups of interest and 3 studies had less than 20 patients in either group ${ }^{17-19}$ ), 8 studies were included in the final meta-analysis ${ }^{7,8,13,20-24}$ (Figure 1). Baseline study characteristics with the MINORS total score are shown in Table 1. A total of 1390 patients were allocated to two groups: a DOAC group of 466 patients and a VKA group of 924 .

\subsection{Outcomes}

\subsubsection{Days in hospital meta-analysis}

5 Studies reported on days in hospital and were included in the meta-analysis ${ }^{13,20-23}$. A significantly longer in-hospital stay was found in the VKA group $(\mathrm{SMD}=-0.22,95 \% \mathrm{CI}-0.42$ to $-0.02, \mathrm{P}=.03$; Table 2$)$. There was no heterogeneity among the studies $\left(\mathrm{I}^{2}=0 \%, 95 \%\right.$ CI 0 to $\left.71.8, \mathrm{P}=.6\right)$. The fixed effects model was therefore applied.

\subsubsection{Posterior epistaxis meta-analysis}

6 Studies reported the rates of posterior epistaxis ${ }^{7,8,13,20,22,23}$ to be significantly more common in the VKA group $(\mathrm{OR}=.39,95 \% \mathrm{CI} .23$ to $.68, \mathrm{P}=.001$; Table 3$)$. The heterogeneity was low $\left(\mathrm{I}^{2}=16.7 \%, 95 \%\right.$ CI 0 to $61.5, \mathrm{P}=.3)$. The fixed effects model was therefore applied.

\subsubsection{Admission rate meta-analysis}

With regard to the rate of admission to hospital as a percentage of all patients seen in the emergency room, data was extracted from 6 studies $7,8,20,21,23,24$. No statistical significance between the groups could be found $(\mathrm{OR}=.76,95 \% \mathrm{CI} .54$ to $1.07, \mathrm{P}=.12$; Table 4$)$. The heterogeneity was low to moderate $\left(\mathrm{I}^{2}=46.03 \%, 95 \%\right.$ CI 0 to $78.63, \mathrm{P}=.1$ ). The fixed effects model was therefore applied.

\subsubsection{Intervention meta-analysis}

7 Studies reported on surgical intervention and/or embolization ${ }^{7,8,13,20-23}$. No statistical significance between the groups could be found ( $\mathrm{OR}=1.29,95 \% \mathrm{CI} .63$ to $2.66, \mathrm{P}=.38$; Table 5$)$. No heterogeneity was detected 
$\left(\mathrm{I}^{2}=0 \%, 95 \%\right.$ CI 0 to $\left.69.3, \mathrm{P}=.5\right)$. The fixed effects model was therefore applied.

3.2.5 Recurrence rate meta-analysis

The recurrence rates were noted in 5 studies $^{7,8,13,21,22}$. The rates were comparable between the groups with no statistically significant difference $(\mathrm{OR}=1.13,95 \% \mathrm{CI} .75$ to $1.7, \mathrm{P}=.57$; Table 6$)$. No heterogeneity was noted $\left(\mathrm{I}^{2}=0 \%, 95 \%\right.$ CI 0 to $\left.41.03, \mathrm{P}=.86\right)$ and the fixed effects model was therefore applied.

\subsubsection{Haemoglobin level meta-analysis}

The haemoglobin value on admission was reported in 5 studies $^{7,13,20,22,23}$. No significant differences between the groups were noted ( $\mathrm{SMD}=-.32,95 \% \mathrm{CI}-.89$ to $.28, \mathrm{P}=.28$; data not shown). The heterogeneity was high $\left(\mathrm{I}^{2}=81.6 \%, 95 \%\right.$ CI 57.2 to $\left.92.1, \mathrm{P}=.0002\right)$. The random effects model was therefore applied.

3.2.7 The need for transfusion meta-analysis

5 Studies reported on the rate of transfusion ${ }^{8,13,20,22,23}$. The need for transfusion was very rare and no statistically significant difference between the groups was found $(\mathrm{OR}=1.34,95 \% \mathrm{CI} .52$ to $3.26, \mathrm{P}=.57$; data not shown). The heterogeneity was very low $\left(\mathrm{I}^{2}=0 \%, 95 \%\right.$ CI 0 to $\left.61.96, \mathrm{P}=.73\right)$. The fixed effects model was therefore applied.

\subsection{Publication bias}

Publication bias was assessed by funnel plots (Tables 2-6, data for haemoglobin value and need for transfusion not shown). The plots were generally symmetrical for all investigated parameters, except for the haemoglobin value. This suggests a low risk of publication bias for the investigated criteria.

\section{Discussion}

We present the first systematic review and meta-analysis of subgroups of epistaxis patients taking OACs by means of data analysis of $466 \mathrm{DOAC}$ and $924 \mathrm{VKA}$ patients. According to our findings, DOAC epistaxis patients appear to have a more favourable outcome when compared to VKA epistaxis patients because they spend notably less time in hospital and have significantly less posterior epistaxis, which is more difficult to control. The heterogeneity and bias of the data used was low, allowing for a pooled analysis.

In order to compare the hospital dynamics and evaluate the disease severity of the two groups of patients, we extracted data from eight included studies with regard to the number of days spent in hospital, the rates of posterior epistaxis, the admission rates for stationary treatment, the intervention rates (including surgery and embolization), and recurrence rates. These indicators were chosen in accordance with the applicable general epistaxis guidelines in the United Kingdom ${ }^{1}$ and United States ${ }^{4}$ as well as the epistaxis guidelines applicable in France for patients taking OACs ${ }^{2}$. In relation to $\mathrm{OAC}$ patients, the guidelines underline the fact that epistaxis patients have a less favourable outcome when taking $\mathrm{OACs}^{25}$, however no comparisons between DOAC and VKA subgroups of patients are noted, strengthening the need for our meta-analysis.

After performing the meta-analysis, statistically significant differences were found for pooled values of days spent in hospital and the rate of posterior epistaxis: all other listed investigated criteria showed no statistically significant differences. All results in this meta-analysis were dichotomous with exception of posterior epistaxis where all the analysed studies demonstrated more posterior epistaxis in VKA patients. This fact caught our attention and led us to search for an understanding and an explanation.

The general rate of posterior epistaxis is $5-10 \%{ }^{26}$. It is more common in older patients and is more difficult to control than anterior epistaxis ${ }^{1}$. The rates of posterior epistaxis in DOAC patients in the included studies were very low with 2 studies reporting an absence of posterior epistaxis in the DOAC group ${ }^{8,13}$, whereas 4 out of 6 studies reported a posterior epistaxis rate of $>10 \%$ in the VKA group of patients. The included studies did not demonstrate a significantly advanced age in the VKA group thus eliminating this factor as an explanation for the discrepancy. One possible explanation is that DOACs and VKAs have different mechanisms of action with respect to the expression of tissue factor (TF) in nasal mucosa and nasal blood vessels adventitia. TF is a coagulation cofactor present on the plasma membrane of certain cells not in 
circulating blood and its expression is tissue dependent ${ }^{27}$. Evidence suggests that the upregulation of TF expression in epithelial mucosa as well as varying TF expressions in the blood vessel adventitia are dependent on vessel calibre ${ }^{27}$. Bearing in mind that posterior epistaxis occurs mostly on the postero-lateral aspect of the middle and inferior turbinate and/or meatus whilst anterior epistaxis occurs in the area of the Kiesselbachii's plexus $^{28}$, we speculate that there is a difference in TF expression in these two areas.

Kawabori et al. investigated patterns of intracerebral haemorrhaging in patients taking OACs and did not find a difference in the bleeding site between DOAC and VKA patients. However blood volume was significantly lower in the DOAC group ${ }^{29}$. In this study, no specific brain vessel was identified as predestined to cause bleeding in the DOAC cohort, contrary to the findings of our meta-analysis of epistaxis. It is interesting to note that the significantly higher rate of posterior epistaxis in the VKA group did not translate into a significantly higher rate of interventions, including operations and/or embolizations.

A possible explanation of the worse outcome in VKA patients could be an inappropriate medication intake. With respect to the papers analysed in the meta-analysis, we were able to extract data on the INR values of VKA patients from 5 of the 8 analysed papers. The reported INR values were generally in the therapeutic range of $2.6-3^{7,13,20,22,23}$. However, Glikson and Send reported that up to one third of their VKA patients were out of their therapeutic INR range ${ }^{20,22}$. The rate of VKA discontinuation was up to $61 \%$ in one study ${ }^{13}$. Various studies demonstrated a strong connection between out-of-range INR and major bleeding events ${ }^{30,31}$. The need for INR monitoring could to some extent be a reasonable explanation for the longer hospital stays in the VKA group.

The recurrence rates found in this meta-analysis were high in both groups, ranging from $10-35 \%$, and with no significant inter-group difference. It is important to identify strategies to avoid recurrence, bearing in mind the age and comorbidities of the patients. The $\mathrm{CH}_{2} \mathrm{ADS}_{2}$-VASc is the favoured risk stratification score for atrial fibrillation patients. A correlation between the $\mathrm{CH}_{2} \mathrm{ADS}_{2}$-VASc Score and epistaxis recurrence has been shown. Furthermore, when the $\mathrm{CH}_{2} \mathrm{ADS}_{2}$-VASc Score reaches or surpasses 7, the recurrence probability surpasses $50 \%$ in OAC epistaxis patients ${ }^{13}$. A recent study demonstrated that after the implementation of an education plan there were significantly less emergency department visits by epistaxis patients taking warfarin ${ }^{32}$. Such strategies should also be considered in DOAC patients. In addition, non-medical alternatives to OACs for patients with atrial fibrillation, such as percutaneous closure of the left atrial appendage (LAA), should be examined ${ }^{33}$. This alternative should be carefully considered after consultation with cardiologists, especially in patients with a $\mathrm{CH}_{2} \mathrm{ADS}_{2}$-VASc Score [?] 7.

Future prospective studies are needed to verify the results of this meta-analysis. In addition, a more accurate understanding of the tendency toward anterior epistaxis in DOAC patients as well as a correlation between out-of-range INR and disease severity is needed.

This study has several limitations that are noteworthy: First of all, we were only able to include 8 studies in the meta-analysis, which could be explained by the fact that DOACs have only been available for a decade. All 8 studies were retrospective and therefore subject to potential bias. We accessed bias according to the MINORS Score and the average value was 14.4, whilst the maximum result was 24. Not all studies reported all of the criteria we sought to investigate and as a result the meta-analysis pro criteria included 5 to 7 studies. Additionally, we cannot guarantee the validity of the results reported, thus possibly affecting the final synthesis.

\section{Conclusion}

According to this systematic review and meta-analysis, epistaxis patients taking DOACs have a more favourable outcome in comparison to VKA patients. DOAC patients have significantly shorter hospital stays and significantly more anterior epistaxis, as opposed to posterior epistaxis, than VKA patients. Large, prospective studies are needed in order to confirm these findings and eliminate potential bias.

\section{References}


1. National ENTTRN. The British Rhinological Society multidisciplinary consensus recommendations on the hospital management of epistaxis. J Laryngol Otol 2017; 131:1142-1156.

2. Escabasse V, Bequignon E, Verillaud Bet al. Guidelines of the French Society of Otorhinolaryngology (SFORL). Managing epistaxis under coagulation disorder due to antithrombotic therapy. Eur Ann Otorhinolaryngol Head Neck Dis 2017; 134:195-199.

3. Garneau JC, Wasserman I, Konuthula N, Malkin BD. Referral patterns from emergency department to otolaryngology clinic. Laryngoscope 2018; 128:1062-1067.

4. Tunkel DE, Anne S, Payne SCet al. Clinical Practice Guideline: Nosebleed (Epistaxis). Otolaryngol Head Neck Surg 2020; 162:S1-S38.

5. Nikolaou G, Holzmann D, Soyka MB. Discomfort and costs in epistaxis treatment. Eur Arch Otorhinolaryngol 2013; 270:2239-2244.

6. Althaus AE, Luske J, Arendt Uet al. Treating epistaxis - who cares for a bleeding nose? A secondary data analysis of primary and secondary care. BMC Fam Pract 2021; 22:75.

7. Runggaldier D, Schindler V, Luo Y, Kleinjung T, Soyka MB. Novel oral anticoagulation treatment is not associated with recurrent or severe epistaxis. Rhinology 2021; 59:219-221.

8. Yaniv D, Zavdy O, Sapir E, Levi L, Soudry E. The Impact of Traditional Anticoagulants, Novel Anticoagulants, and Antiplatelets on Epistaxis. Laryngoscope 2021.

9. Kallenbach M, Dittberner A, Boeger Det al. Hospitalization for epistaxis: a population-based healthcare research study in Thuringia, Germany. Eur Arch Otorhinolaryngol 2020; 277:1659-1666.

10. Althaus AE, Arendt U, Hoffmann Fet al. [Epistaxis and anticoagulation therapy: an analysis based on health insurance data from Lower Saxony]. HNO 2021; 69:206-212.

11. Hindricks G, Potpara T, Dagres Net al. 2020 ESC Guidelines for the diagnosis and management of atrial fibrillation developed in collaboration with the European Association for Cardio-Thoracic Surgery (EACTS). Eur Heart J 2021; 42:373-498.

12. Ortel TL, Neumann I, Ageno Wet al. American Society of Hematology 2020 guidelines for management of venous thromboembolism: treatment of deep vein thrombosis and pulmonary embolism. Blood Adv 2020; 4:4693-4738.

13. Stankovic P, Georgiew R, Frommelt Cet al. Shorter hospital stays in epistaxis patients with atrial fibrillation when taking rivaroxaban or apixaban versus phenprocoumon. J Thromb Thrombolysis 2019; 47:384-391.

14. Page MJ, McKenzie JE, Bossuyt PMet al. The PRISMA 2020 statement: an updated guideline for reporting systematic reviews. BMJ 2021; 372:n71.

15. FDA Approval Dabigatran. Available at: https://www.accessdata.fda.gov/drugsatfda_docs/nda/2010/022512orig1s000toc.cfm. Accessed 26.04.2021.

16. Slim K, Nini E, Forestier D, Kwiatkowski F, Panis Y, Chipponi J. Methodological index for nonrandomized studies (minors): development and validation of a new instrument. ANZ J Surg 2003; 73:712716.

17. L'Huillier V, Badet C, Tavernier L. Epistaxis complicating treatment by anti-vitamin K and new oral anticoagulants. Eur Ann Otorhinolaryngol Head Neck Dis 2018; 135:231-235.

18. Gokdogan O, Akyildiz I, Sayin BY, Okutucu S, Tanalp AC, Arslan N. The Rate of Epistaxis Incidence in New-Generation Anticoagulants and Perioperative Approach in Otorhinolaryngological Practices. J Craniofac Surg 2017; 28:e178-e182. 
19. García Callejo FJ, Bécares Martínez C, Calvo González J, Martínez Beneyto P, Marco Sanz M, Marco Algarra J. Epistaxis and dabigatran, a new non-vitamin K antagonist oral anticoagulant. Acta Otorrinolaringol Esp 2014; 65:346-354.

20. Send T, Bertlich M, Horlbeck Fet al. Management and outcome of epistaxis under direct oral anticoagulants: a comparison with warfarin. Int Forum Allergy Rhinol 2019; 9:120-124.

21. Buchberger AMS, Baumann A, Johnson Fet al. The role of oral anticoagulants in epistaxis. Eur Arch Otorhinolaryngol 2018; 275:2035-2043.

22. Glikson E, Chavkin U, Madgar Oet al. Epistaxis in the setting of antithrombotic therapy: A comparison between factor Xa inhibitors, warfarin, and antiplatelet agents. Laryngoscope 2019; 129:119-123.

23. Sauter TC, Hegazy K, Hautz WEet al. Epistaxis in anticoagulated patients: Fewer hospital admissions and shorter hospital stays on rivaroxaban compared to phenprocoumon. Clin Otolaryngol 2018; 43:103-108.

24. Davies T, Alatsatianos A, Slim MAM, Royce W, Whymark A. The impact of frailty on epistaxis admission, a retrospective cohort study. Clin Otolaryngol 2021.

25. Sethi RKV, Kozin ED, Abt NB, Bergmark R, Gray ST. Treatment disparities in the management of epistaxis in United States emergency departments. Laryngoscope 2018; 128:356-362.

26. Cooper SE, Ramakrishnan VR. Direct cauterization of the nasal septal artery for epistaxis. Laryngoscope $2012 ; 122: 738-740$.

27. Fleck R.A., Rao M., Rapaport S., N. V. Localization of human tissue factor antigen by immunostaining with monospecific, polyclonal anti-human tissue factor antibody. Thrombosis Research 1990; 59:421-437.

28. Thornton MA, Mahesh BN, Lang J. Posterior epistaxis: identification of common bleeding sites. Laryngoscope 2005; 115:588-590.

29. Kawabori M, Niiya Y, Iwasaki Met al. Characteristics of Symptomatic Intracerebral Hemorrhage in Patient Receiving Direct Oral Anticoagulants: Comparison with Warfarin. Journal of Stroke and Cerebrovascular Diseases 2018; 27:1338-1342.

30. Nelson WW, Wang L, Baser O, Damaraju CV, Schein JR. Out-of-range INR values and outcomes among new warfarin patients with non-valvular atrial fibrillation. Int J Clin Pharm 2015; 37:53-59.

31. Merli GJ, Tzanis G. Warfarin: what are the clinical implications of an out-of-range-therapeutic international normalized ratio? J Thromb Thrombolysis 2009; 27:293-299.

32. Neshewat J, Wasserman A, Alexandris-Souphis Cet al. Reduction in epistaxis and emergency department visits in patients taking warfarin after implementation of an education program. Thromb Res 2021; 199:119122 .

33. Jame S, Barnes G. Stroke and thromboembolism prevention in atrial fibrillation. Heart 2020; 106:10-17.

\section{Appendix}

Figure1. Flow chart of study selection according to PRISMA guidelines

Table1. Included studies, number of patients and investigated criteria

Table2. Days in hospital Meta-Analysis

Table3. Posterior Epistaxis Meta-Analysis

Table4. Admission rate Meta-Analysis

Table5. Intervention (surgery and/or embolization) Meta-Analysis

Table6. Recurrence rate Meta-Analysis 


\section{Hosted file}

figure1.docx available at https://authorea.com/users/437961/articles/539277-direct-oralanticoagulants-versus-vitamin-k-antagonists-in-epistaxis-patients-a-systematic-reviewand-meta-analysis

\section{Hosted file}

table1.docx available at https://authorea.com/users/437961/articles/539277-direct-oralanticoagulants-versus-vitamin-k-antagonists-in-epistaxis-patients-a-systematic-reviewand-meta-analysis

\section{Hosted file}

table2.docx available at https://authorea.com/users/437961/articles/539277-direct-oralanticoagulants-versus-vitamin-k-antagonists-in-epistaxis-patients-a-systematic-reviewand-meta-analysis

\section{Hosted file}

table3.docx available at https://authorea.com/users/437961/articles/539277-direct-oralanticoagulants-versus-vitamin-k-antagonists-in-epistaxis-patients-a-systematic-reviewand-meta-analysis

\section{Hosted file}

table4.docx available at https://authorea.com/users/437961/articles/539277-direct-oralanticoagulants-versus-vitamin-k-antagonists-in-epistaxis-patients-a-systematic-reviewand-meta-analysis

\section{Hosted file}

table5.docx available at https://authorea.com/users/437961/articles/539277-direct-oralanticoagulants-versus-vitamin-k-antagonists-in-epistaxis-patients-a-systematic-reviewand-meta-analysis

\section{Hosted file}

table6.docx available at https://authorea.com/users/437961/articles/539277-direct-oralanticoagulants-versus-vitamin-k-antagonists-in-epistaxis-patients-a-systematic-reviewand-meta-analysis 